\title{
RESEARCHARTICLE
}

\section{FORMULATION, CHARACTERIZATION AND PHARMACOKINETIC EVALUATION OF NARINGENIN- LOADED GASTRORETENTIVE MUCOADHESIVE POLYMERIC NANOS YSTEM FOR ORAL DRUG DELIVERY}

\author{
P Suseela, SD Saras wathy \\ Department of Bio med ical Science, School of Bas ic Medical Sciences, Bharathidasan University, Tiruchirappalli \\ 620024, Tamil Nadu, India \\ "Corresponding Author's Email: sd.saraswathy@gmail.com
}

Received 24 Jan 2015; Review Completed 14 March 2015; Accepted 14 March 2015, Available online 15 March 2015

\begin{abstract}
Nanop articles mediated oral drug delivery system has attracted great attention in pharmacology to attain improved therapeutic index of chemotherapeutic drugs. In the present study, naringenin (NRG) - loaded gastroretentive mucoadhesive polymeric nanoparticles has been designed and investigated its application in oral drug delivery system. Naringenin loaded gastroretentive mucoadhesive polymeric nanoparticles (NMPN) were prepared by thermal decomposition mediated nanoprecip itation method. Formulated NMPN were characterized and investigated for its mucoadhesive properties by swelling and in vitro mucoadhesion studies. The results indicated that the formulated NMPN were in the size of $66 \pm 7 \mathrm{~nm}$ by dynamic light scattering analys is and the maximum drug loading efficiency was observed around $94.6 \pm 0.28 \%$. Further the drug release studies showed that NMPN exhibited sustained drug release in stimulated gastric fluid $(\mathrm{SGF} ; \mathrm{pH}=1.2)$. Pharmacokinetic studies revealed that the formulated NMPN are highly biocompatible and showed enhanced therapeutic efficacy compared to free naringenin. The overall findings demonstrated that the gastroretentive mucoadhesive poly meric nanoparticles can act as a promising candidate with gastroprotective applications in biomedical research.
\end{abstract}

Keywords: Naringenin, drug delivery, stimulated gastric fluid, mucoadhesive

\section{INTRODUCTION}

In recent years, oral controlled drug delivery has attracted great attention in pharmaceutical field in order to attain improved therapeutic advantages, such as short elimination half life, systemic toxicities and patient compliance so forth. ${ }^{1}$ Nanoparticles mediated drug delivery reduces the frequency of dosing in therapeutic conditions by sustained drug release and maintains its effective drug concentration in the systemic circulation over a longer period of time. $^{2}$ Even though, the systemic drug delivery of nanosystems is in market, oral drug delivery is important in various clinical conditions, particularly gastro intestinal tract disorders. More recently, the pursuit and exploration of devices designed to be retained drugs in the upper part of the gastrointestinal tract (GIT) has advanced consistently in terms of technology and diversity, enco mpass ing a variety of biosystems and devices like floating systems, swelling-expanding systems, bioadhes ive system, modified- shape syste m etc. ${ }^{3}$ Gastric retention has advantages in delivering of drug with narrow absorption in the upper part of GIT. Also, longer residence time in the stomach could be advantageous for local action in the GIT, particularly treatment of peptic ulcer dis eases.
Gastroretentive drug delivery is one of the site specific deliveries for che motherapeutic drugs either at stomach or at intestine level. ${ }^{4}$ It is obtained by retaining dosage form into stomach and drug is being released at controlled manner to specific site either in stomach, duodenum and in testine. Naring en in (NRG, 4, 5, 7- trihydroxy flavanone), a naturally occurring flavonoid, and aglycone of naring in, is widely present in citrus fruits, tomatoes, cherries, grapefruit and cocoa. ${ }^{5,6,7,8,9}$ It is well known for various biological actions, such as antioxidant, anti- inflammatory, anti-ulcer, as well as anti-carcinogenic effects..$^{10,11}$ The drug, Yet it suffers from biopharmaceutical restrictions due to its poor water solubility which results in poor absorption and short half life about 2 hours. ${ }^{12,13}$ Also NRG has been reported to have short drug residence time in the stomach. ${ }^{14}$ So it is necessary to develop gastroretentive delivery system to maintain its integrity.

In the present study was an attempt to design and formulate NRG- loaded gastroretentive mucoadhesive polymeric nanoparticles using synthetic polymers such as Eudragit E 100 (EE 100) and polyvinyl alcohol (PVA). The 
physicochemical characterization of NMPN was carried by dynamic light scattering (DLS), zeta potential, loading efficiency, drug loading content, fourier transform infrared spectroscopy (FTIR), nuclear magnetic resonance spectroscopy $\left({ }^{1} \mathrm{H}\right.$ NMR) and field emission transmission electron microscopy (FE- TEM) studies. The mucoadhes ive properties were determined by swelling and in vitro mucoadhesion studies. Also, drug release in stimulated gastric and intestinal fluids has been assessed. The in vivo pharmacokinetic pattern of NMPN was also studied in $\mathrm{W}$ istar rats.

\section{MATERIALS AND METHODS}

Eudragit $^{\circledR}$ E 100 (EE 100) was obtained from Evonik Industries, Mumbai, India. Naringenin (NRG) was purchased from Sigma- Aldrich Chemicals, St. Louis, MO., Mumbai, India. While other fine chemicals, reagents and solvents used for the experiments were of analytical grade, purchased from Merck Ltd., Mumbai, India. Milli Q water was used in all experiments.

\section{Formulation of NMPN: ${ }^{15,16,17}$}

NMPN were formulated using a nanoprecipitation technique with slight modifications. The internal organic phase composed of $50 \mathrm{mg}$ NRG and $500 \mathrm{mg}$ EE $100 \mathrm{in} 25 \mathrm{ml}$ of ethanol. This internal solution was quickly injected into the $75 \mathrm{ml}$ external aqueous solution containing $500 \mathrm{mg}$ of PVA, and then the solutions were stirred. Then the emulsion was ripened at $100{ }^{\circ} \mathrm{C}$ for an hour by introducing the thermal decomposition and cooled in an appropriate manner. The reaction mixture was purified by centrifugation at $6000 \mathrm{rpm}$ to remove small particles. The precipitate was main ly composed of nanoparticles. It was lyophilized and stored for further use.

\section{Physicochemical char acterization Techniques:}

\section{DLS analysis of NMPN:}

Measurement of particle size, polydispersity index of the free NRG and NMPN was performed by Photon Correlation Spectroscopy known as Dynamic Light Scattering (Nano- ZS90 - Malvern Instruments). Each sample was appropriately diluted 10 fold with ultra purified water and measured at $25^{\circ} \mathrm{C}$ and $90^{\circ}$ scattering angle, recorded for $180 \mathrm{~s}$. Each value was measured in triplicate. The results were showed as mean $\pm \mathrm{SD}$ distribution. Surface charge of the NMPN were also characterized with Zeta potential $(\zeta)$ using a Malvern Nano- ZS90. The measurements were done using an aqueous dip cell in an automatic mode by placing diluted samples (with ultra-purified water) in the capillary measurement cell and cell position was adjusted.

${ }^{\mathbf{1}}$ H Nuclear Magnetic Res onance ( ${ }^{\mathbf{1}}$ H NMR): ${ }^{18}$

The ${ }^{1} \mathrm{H}$ NMR spectra of free NRG and NMPN were recorded using a JEOL Alpha 400 spectrometer. An amount of $50 \mathrm{mg}$ of each sample was dissolved in $1.0 \mathrm{ml}$ of DMSO-d6 and each sample was measured and recorded in triplicate.

Fourier Transform Infrared S pectroscopy (FTIR):
Infrared spectra were obtained using a Perkin Elmer FTIR spectrometer. In order to collect the spectra, a small amount of the compound was used by compression in $\mathrm{KBr}$ tablets. The IR spectra were obtained in the spectral region $400-4000 \mathrm{~cm}-1$ using resolution $4 \mathrm{~cm}^{-1}$ and 10 co-added scans. The spectrum of the $\mathrm{KBr}$ pellet was used as background. The spectra presented were baseline corrected and normalized.

\section{Morphology in Electron Microscopy:}

The morphology of the NMPN was observed using field emis sion Scanning electron mic roscopy (FETEM) (JSM 2100, JEOL, Japan). One drop of properly diluted nanoparticles suspension was placed on a silicon substrate and air dried under ambient condition to study the morphology at various magnifications.

\section{Loading Efficiency of NMPN: ${ }^{19}$}

Loading efficiency of $\mathrm{NRG}$ was determined after the twice centrifugation at $12,000 \mathrm{rpm}$ and $4{ }^{\circ} \mathrm{C}$ for $25 \mathrm{~min}$. The amount of free NRG was determined in clear supernatant by Schimadzu UV 1800 spectrophotometer with the detection wavelength of 262 $\mathrm{nm}$. The following equation were applied to calculate the loading efficiency $(\%)=\left[\left(\mathrm{W}_{\mathrm{O}}-\mathrm{W}_{\mathrm{t}}\right) / \mathrm{W}_{\mathrm{O}}\right] \mathrm{X} 100$. $\mathrm{W}_{\mathrm{O}}$ and $\mathrm{W}_{\mathrm{t}}$ are the weight of initial concentration of NRG and that of the total amount of NRG detected in supernatant after twice centrifugation respectively. Each sample was as sayed in triplicate.

\section{Evaluation of Mucoadhesi ve property:}

Swelling Study: ${ }^{20}$

The swelling studies on NMPN were performed with $0.1 \mathrm{~N} \mathrm{HCl}(\mathrm{pH} 1.2)$ and phosphate buffer ( $\mathrm{pH}$ 7.4). In brief, $100 \mathrm{mg}$ of NMPN accurately weighed and immersed in $0.1 \mathrm{~N} \mathrm{HCl}$ and phosphate buffer, and kept for 24 hrs. The swelling index (SI) was calculated by using the following formula: $\mathrm{SI}=(\mathrm{Wi}-$ $\mathrm{Ws} / \mathrm{Ws}$ ) X 100. Where SI= percentage of swelling of nanoparticles, $\mathrm{Wi}=$ in itial weight of nanoparticles, and $\mathrm{Ws}=$ weight of nanoparticles after swelling.

In-vitro mucoadhesion studies: ${ }^{21}$

Naringenin- loaded gastroretentive mucoadhesive polymeric nanoparticles were immersed in a $50 \mathrm{ml}$ glass beaker at $37^{\circ} \mathrm{C}$ containing a $0.1 \mathrm{~N} \mathrm{HCl}$ (pH 1.2). Fresh rat gastric mucosa was cut into $2 \mathrm{X} 3 \mathrm{~cm}$ and was placed on nanoparticles surface. Gastric mucosa was removed after $30 \mathrm{~min}$ and mucosa with the attached nanoparticles were removed and the remaining particles on the glass beaker were dried at $60{ }^{\circ} \mathrm{C}$ till constant weight. Percent of adhered nanoparticles (AN) was estimated using the following equation: $\mathrm{AN}(\%)=$ (Wo - Wr/ Wo) X 100. Where, Wo is the initial weight of nanoparticles and $\mathrm{Wr}$ the remained unattached weight of nanoparticles.

\section{In vitro release studies:}

The in vitro drug release studies were conducted in stimulated gastric fluid $(\mathrm{pH}$ 1.2) and stimulated intestinal fluid $(\mathrm{pH}$ 6.8). An appropriate amount of NMPN was placed in a beaker containing 50 $\mathrm{ml}$ of simulated gastric fluid and intestinal fluid at 37 
${ }^{\circ} \mathrm{C}$ with shaking conditions. $0.1 \mathrm{ml}$ of fluid was withdrawn at each time point periodically and replaced with the same volume of fresh fluid and the amount of drug was quantified by HPLC analysis.

\section{Evaluation of Phar macokinetic Profile:}

Animals and Dosing: All the animal study protocols were duly approved by the Institutional Animal Ethics Committee, India. Male Wister strain rats weighing 200-250 g were maintained in controlled room temperature $\left(20^{\circ} \mathrm{C} \pm 2^{\circ} \mathrm{C}\right)$ and relative humidity $(60 \% \pm$ $10 \%$ ) on $12 \mathrm{hrs}$ light/dark cycle. Water and commercial laboratory complete food for rats were available $a d$ libitum. They were acclimatized to this environment for 7 days before receiving experimental treatment. The animals were randomly distributed into two groups each containing six animals. Different groups of animals received oral dose of free NRG $(20 \mathrm{mg} / \mathrm{kg})$ and NMPN $(20 \mathrm{mg} / \mathrm{kg}$ ). Blood samples (appro ximately $0.5 \mathrm{ml})$ were collected at periodic time intervals $(0,4,8,12,16,20$ and $24 \mathrm{hrs}$ ) from the retro- orbital plexus. After each sampling, $1 \mathrm{ml}$ of dextrose- normal saline was administered orally to prevent changes in the central compartment volume and electrolytes. Plasma was separated by centrifuging the blood samples at 10,000 $\mathrm{rpm}$ for $10 \mathrm{~min}$ at $4{ }^{\circ} \mathrm{C}$ and kept at $-80{ }^{\circ} \mathrm{C}$ until analyzed.

\section{Determination of naringenin in rat plasma by HPLC:}

Naringenin in rat plasma was analyzed by HPLC using a mixture of acetonitrile and $2 \%$ acetic acid in a volume ratio of $51: 49(\mathrm{v} / \mathrm{v})$ as the mobile phase at a flow rate of $1.0 \mathrm{ml} \cdot$ minute -1 . For the measurement of plasma NRG, $50 \mu 1$ of internal standard solution (hesperitin, $4 \mu \mathrm{g} \cdot \mathrm{ml}-1$ in ethanol) was added to $0.2 \mathrm{ml}$ plasma. After vortex mixing for $1 \mathrm{~min}, 500 \mu \mathrm{l}$ of anhydrous diethyl ether was added and again vortexmixed for $1 \mathrm{~min}$. After centrifugation at $10,000 \mathrm{rpm}$ for $10 \mathrm{~min}$, the supernatant was withdrawn and evaporated under a light stream of nitrogen at room temperature. The residue was dissolved in $100 \mu$ lof the mobile phase and again centrifuged at $10,000 \mathrm{rpm}$ for $10 \mathrm{~min}$. $20 \mu \mathrm{l}$ of the supernatant was then injected for HPLC analysis. Quantification was based on the peak area ratio $R$ (ACUR/AEMO). The calibration curve obtained was $\mathrm{R}$ $=0.0023 \mathrm{C}+0.0335(\mathrm{r}=0.9998, \mathrm{n}=5)$, with $\mathrm{a}$ correlation coefficient 0.999 . The pharmacokinetic parameters of free NRG as well as NMPN groups were calculated by using noncompartmental method. The area under the curve and the mean residence time were determined by standard methods applying the linear trapezoidal rule.

\section{STATISTICAL ANALYS IS}

GraphPad Prism 5 software was used for statistical analys is (GraphPad Software Inc., San Diego, USA). Data are expressed as mean \pm SD. Data were analyzed by one-way analysis of variance (ANOVA) followed by the Student-Newman-Keul multiple comparison test. Differences were considered significant at $P<0.05$.

\section{RES ULTS AND DISCUSS ION:}

NMPN were formulated by a simple nanoprecipitation method with thermal decomposition approaches. Nanoprecipitation is a simplistic and mild process technique for the preparation of nano scale compounds and it was one of the most superior to other encapsulation methods. ${ }^{22}$ During the particle synthes is both internal phases of NRG and EE 100 are hydrophobic in nature interacts each other and form a suspension. These internal phase suspension was introduced into external aqueous phase and produce interfacial tension to form stronger outer surface with the help of PVA as a stabilizer. Nanoprecipitation was conducted through the precipitation of a performed polymer from an organic solution which was previously solubilized, with the diffusion of this organic solvent into the aqueous medium. In the aqueous phase, the polymers get insoluble, precipitating immediately leading to the formation of nanoparticles. ${ }^{23}$ In addition to that under thermal decomposition by means of the mixture face above melting temperature and it was cooled very slowly to form crystals which results in the formulation of cubic particles..$^{24,25,26}$

Figure 1a showed the mean particle size distribution of the formulated nanoparticles. Nanoparticles size is a useful parameter since its affects the drug loading and release. The size distribution of the formulated NMPN was smaller with narrow distributions. The magnitude of the zeta potential on the NMPN was relatively high due to their stronger electrostatic repulsion that inhibits the particle aggregation $^{27}$ that showed in Figure $1 b$. 
(a)

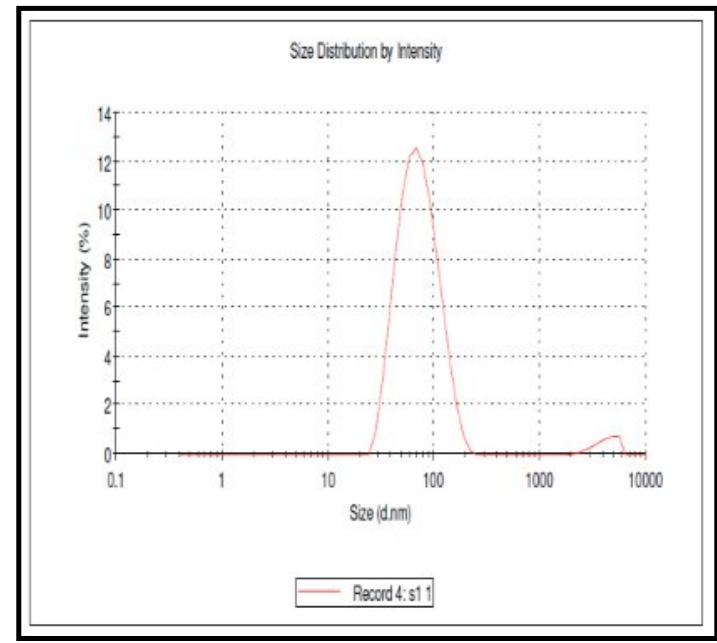

(b)

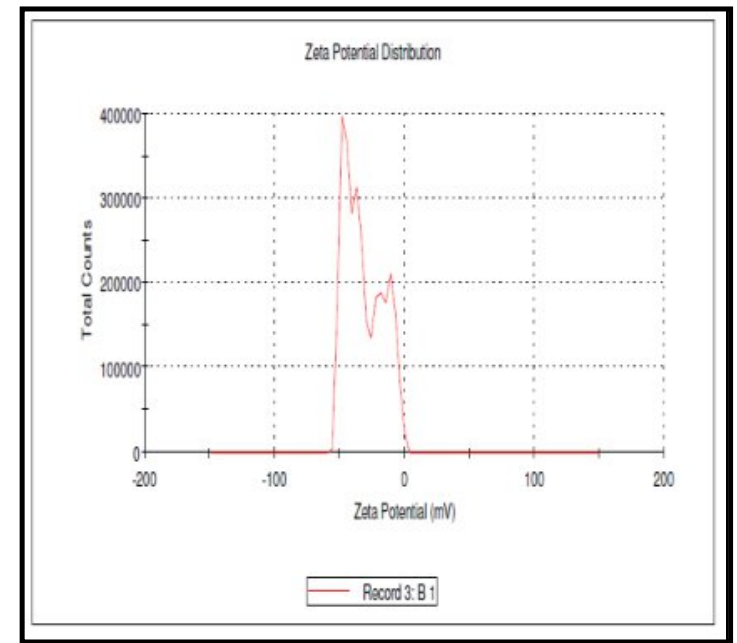

Figure 1: The Graphical representation of (a) mean particle size; (b) zeta potential of formu lated naringenin- loaded gastroretentive mucoadhesive polymeric nanoparticles.

Formulated NMPN were characterized by ${ }^{1} \mathrm{H}$ NMR and FTIR, the hydrogen bonding of free NRG as well as NMPN were showed in Figure 2. Hydrogen bonding formation can be accounted by chemical shift changes in compound. ${ }^{2829}$ The spectrum of free NRG showed protons on aromatic groups ranging from $\mathrm{H} 6-$ H8 (6.77- 6.79ppm) and strong intra molecular hydrogen bonding at $12.14 \mathrm{ppm}$. Where NMPN showed that the aromatic protons were shifted and the intra mo lecular hydrogen bonding disappeared.

The spectrum of NMPN showed protons on aromatic group from $\mathrm{H} 6-\mathrm{H} 8$ was shifted to about
$0.1 \mathrm{ppm}$. In frared spectroscopy was used to study the interactions between the drug and the polymers that showed in Figure 3. Functional characteristics peak for drug in the region of $3849 \mathrm{~cm}^{-1}$. An FTIR spectrum of NMPN does not have a characteristic peak indicating that drug had been loaded into nanoparticles. NMPN spectra did not show any changes in characteristic peaks for EE-100 and PVA in the region of $1390-1370 \mathrm{~cm}^{-1}$ and $1407 \mathrm{~cm}^{-1}$ respectively, its indicating that polymer and stabilizer inter connected with outer layer in nanostructure.

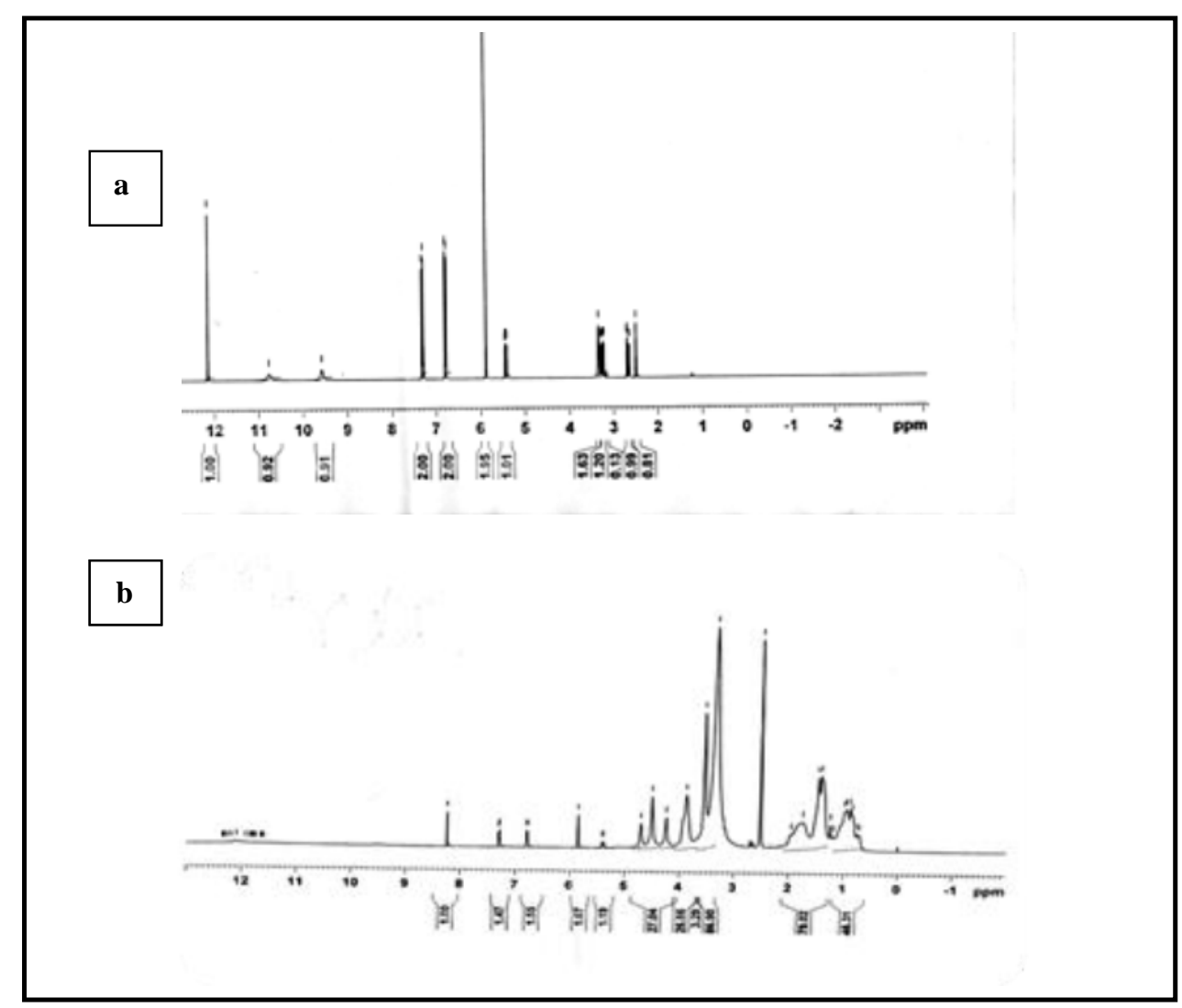

Figure 2: ${ }^{1} \mathrm{H}$ NMR spectrum of (a) free NRG and (b) NMPN dissolved in DMSO- $\mathrm{d}_{6}$. 


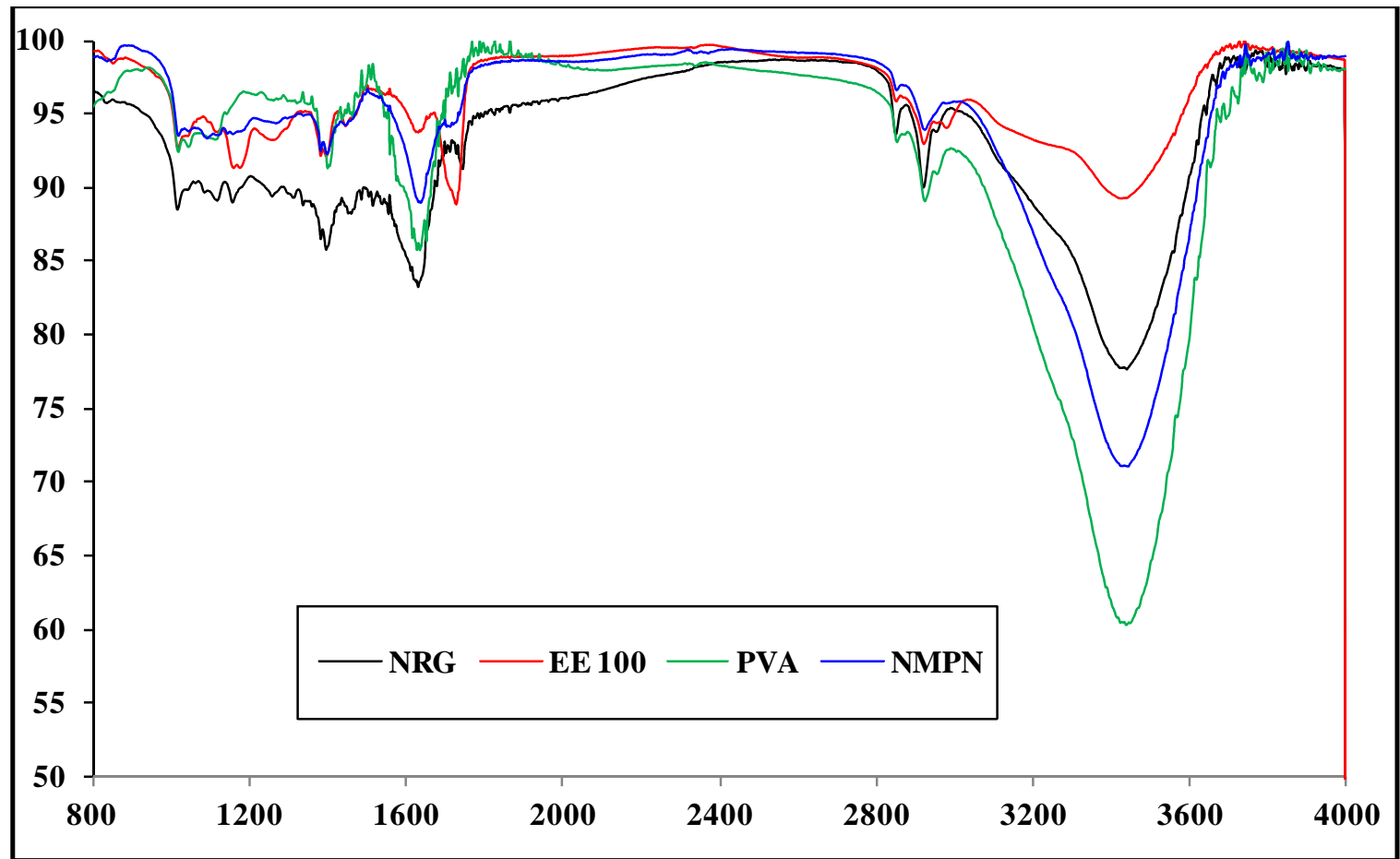

Figure 3: Infrared spectrum of indiv idual co mpounds NRG, EE 100, PVA and NMPN.
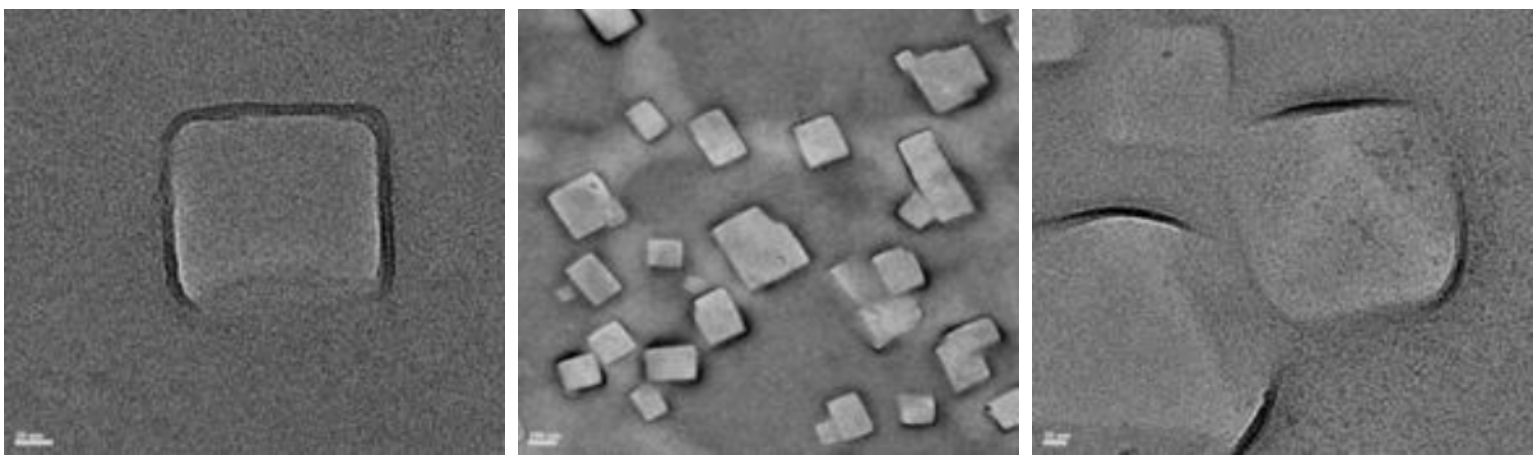

Figure 4: FE- TEM mic rograph of formu lated NMPN at various magnifications.

Nanoparticles morphology was shown in Figure 4. The particles had a cubic morphology with a smooth surface with no polymeric aggregates. Particles are smaller in size, among most of them are similar in dimensions. Hydrodynamic process takes place during the particle preparation which leads to the higher loading efficiency of NRG in surrounding aqueous phase. When adjusting the proportion of stabilizer (PVA) in aqueous phase that influencing the loading efficiency of NRG. The possible reason was the hydrophobic nature of EE 100 interpenetrates into the hydrophilic nature of PVA during particle preparation and remained that trapped to the matrix structure.

The loading efficiency of NMPN was showed in Table 1. Hydrodynamic process takes place during the particle preparation which leads to the higher encapsulation of NRG in surrounding aqueous phase. The hydrophobic nature of EE 100 interpenetrates into the hydrophilic nature of PVA during particle preparation and remained that trapped to the matrix structure.

Table 1: Determination of mean particle size, zeta potential, poydispersity index (PDI) and loading efficiency of blank Nanoparticles (NP) and formu lated NMPN (mean $\pm \mathrm{SD}, \mathrm{n}=3$ ).

\begin{tabular}{|l|l|l|l|l|}
\hline Nanoparticles & Size $(\mathrm{nm})$ & Zeta Potential (mv) & PDI & Loading effic iency (\%) \\
\hline Blank NP & 62.4 & $-25 \pm 1.3$ & $0.20 \pm 0.027$ & -- \\
\hline NMPN & 66.7 & $-34 \pm 0.64$ & $0.22 \pm 0.019$ & $89.4 \pm 2.41$ \\
\hline
\end{tabular}


The $\mathrm{pH}$ is one of the major factors that significantly influence the swelling behavior of a system. The $\mathrm{p}^{\mathrm{H}}$ responsive system has been most frequently used to design controlled release formulations for oral administration of drug, which remains the most clinically acceptable way of drug delivery. ${ }^{30}$ The present study the effect of $\mathrm{pH}$ on the swelling behavior of NMPN was investigated at $\mathrm{pH}$ of 1.2 and 7.4 which represents the $\mathrm{pH}$ conditions of stomach and blood respectively. The swelling results are shown in Figure 5 which indicates a significant faith of swelling behavior of the NMPN on different $\mathrm{pH}$ concentrations. The results revealed at $\mathrm{pH} 1.2$, a higher swelling ratio was found when compared to $\mathrm{pH} 7.4$ which is due to the mucoadhesive polymer used for the preparation of nanoparticles. Mucoadhesive studies had been done in order to ass ess the adhesion of NMPN on gastric mucosal membrane. NMPN shows satisfactory adhesion property which is due to the mucoadhesive polymer used. Mucoadhesive property of EE 100 are mostly dependent on the ionic interaction of positively charged amine group of EE 100 with negatively charged ions present within mucus membrane of sulfonic acid and sialic acid residues.

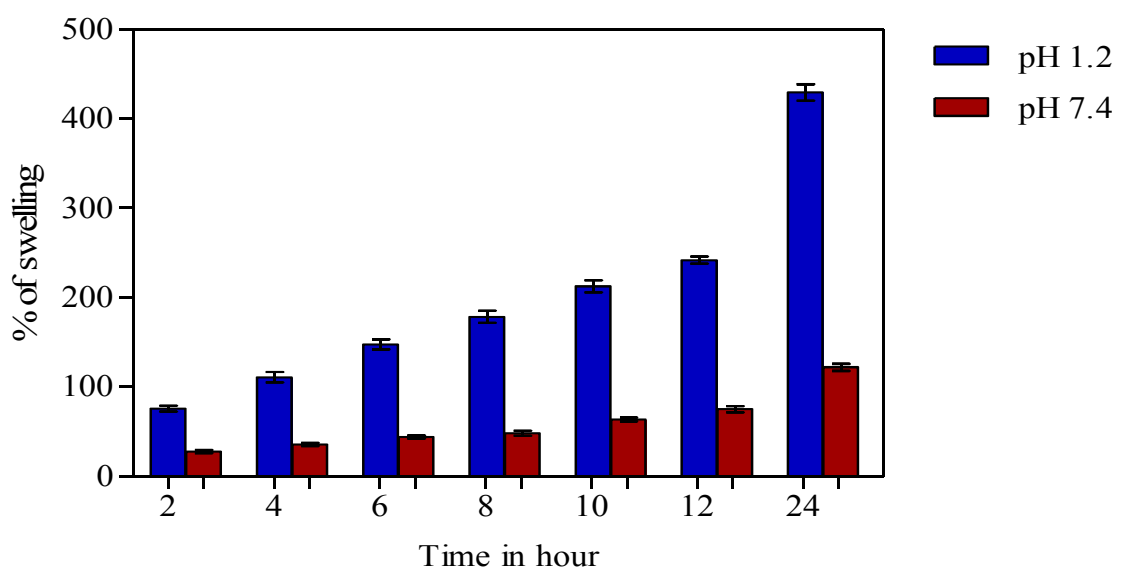

Figure 5: Swelling profile of NMPN at $\mathrm{pH} 1.2$ and 7.4. Indicated values are the mean of three measurements (mean \pm $\mathrm{SD}, \mathrm{n}=3)$.

The release profile of NMPN in stimulated gastric and intestinal fluids showed in Figure 6 (a \& b). Eudragit E 100 is a copolymer of methacrylic acid and ethyl acrylate that soluble below the $\mathrm{pH}$ of 4.5 . It should therefore be possible to design polymeric system that loaded and protect bioactive compounds within acidic environments (site specific, stomach). We therefore investigated the release of NRG from NMPN. In NMPN the drug was released up to $24 \mathrm{hrs}$ in stimulated gastric fluid thus indicating long acting controlled release with high precision. Therefore the prolonged release pattern of NRG which might contributes to reduce the frequent dose and enhances the drug absorption. ${ }^{31}$
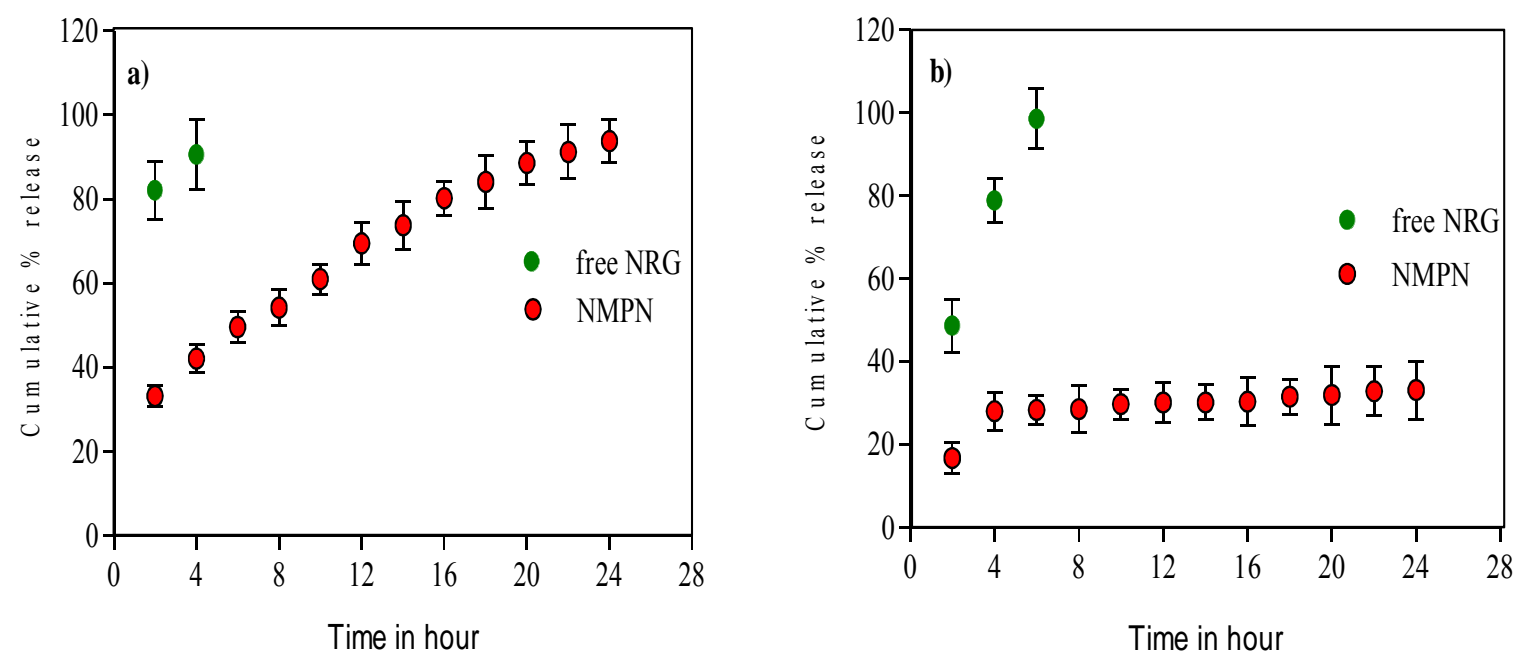

Figure 6: In vitro release profile of free NRG and NMPN in (a) stimulated gastric fluid (pH 1.2) and (b) stimu lated intestinal flu id ( $\mathrm{pH}$ 6.8) at various time intervals (mean $\pm \mathrm{SD}, \mathrm{n}=3$ ). 


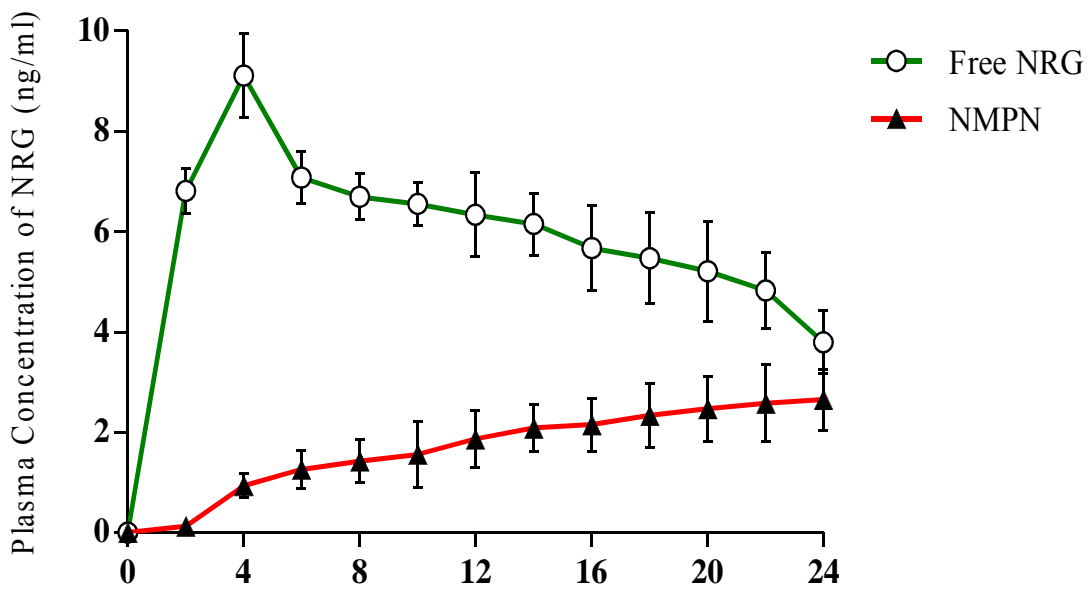

Time in hour

Figure7: Plas ma concentration- time profile of free NRG and NMPN after oral administration of $20 \mathrm{mg} / \mathrm{kg}$ body weight in rats (mean $\pm \mathrm{SD}, \mathrm{n}=6$ ).

The plasma concentration time profile of free NRG and NMPN showed in Figure 7. Main pharmacokinetics parameters in plasma as well as stomach tissue homogenate following a single oral administration of $20 \mathrm{mg} / \mathrm{kg}$ body weight in rats showed in Table 2. NMPN is well absorbed due to the controlled release in acidic media without degradation of the drug when compared with the free NRG. The pharmacokinetic parameters AUC and MRT show significant differences between free NRG and NMPN in plasma and stomach tissue homogenate. Longer residence time in stomach tissue homogenate allows more active components to penetrate through the gastric mucus membrane and to be absorbed above the absorption zone. ${ }^{31}$ Therefore, the formulated NMPN are suitable for stomach specific delivery.

Table 2: Main pharmacokinetics parameters following a single oral dose administration of free NRG and NMPN 20 $\mathrm{mg} / \mathrm{kg}$ body weight in rats (mean $\pm \mathrm{SD}, \mathrm{n}=6$ ).

\begin{tabular}{|c|c|c|}
\hline Groups & AUC (ng h/ml) & $\overline{\text { MRT }}($ \\
\hline \multicolumn{3}{|l|}{$\underline{\text { Plasma }}$} \\
\hline Free NRG & $4523.71 \pm 21.5$ & $3.85 \pm 0.84$ \\
\hline NMPN & $2158.29 \pm 17.3$ & $2.10 \pm 0.52$ \\
\hline \multicolumn{3}{|l|}{$\underline{\text { Stomach Tissue }}$} \\
\hline Free NRG & $2710.48 \pm 28.4$ & $1.80 \pm 0.35$ \\
\hline NMPN & $9776.53 \pm 25.6$ & $16.58 \pm 1.08$ \\
\hline
\end{tabular}

\section{CONCLUS ION}

The naringenin- loaded gastroretentive mucoadhesive polymeric nanoparticles has been formulated by a facile nanoprecipitation method under thermal decomposition. The formulated NMPN have been smaller in size with higher stability and higher drug loading efficiency. The morphology was evidenced by FE-TEM and functional characteristics were confirmed by FTIR and ${ }^{1} \mathrm{H}$ NMR. The Sustained drug release of NRG was prolonged up to $24 \mathrm{hrs}$ in stimulated gastric fluid. Swelling and mucoadhesion studies demonstrated that NMPN exhibited good mucoadhesive in nature. Thus the results of the present study were suggested that the NMPN was found to be a sustained drug delivery vehicle with prolonged release when injected in the form of oral delivery. Pharmacokinetic fate was investigated in NMPN, they have a site specific delivery in stomach due to increased AUC and residence time as compared to free NRG. Further, the toxicity will be assessed in vitro and in vivo tumor models, in order to explore its therapeutic efficacy in the field of biomed ical application.

\section{ACKNOWLEDGEMENT}

The authors declare that there are no conflicts of interest. 


\section{REFERENCES}

1. Nokhodchi A, Raja S, Patel P, Asare- Addo K, The Role od oral controlled release matrix tablets in drug delivery systems. Bioimpacts. 2012; 2(4) 178- 87.

2. Casettari L, Cespi M, Castagnino E, Evaluation of dibutyrylchitin as new excipient for sustained drug release. DrugDevIndPharm. 2012; 38(8) 979-84 .

3. Kumar K, Dhawan N, Sharma H, Vaidya S, Vaidya B, Bioadhesive polymers: novel tool drug delivery. Artif Cells Nanomed Biotechnol. 2014; 42 (4) 274-83.

4. Sarparanta MP, Bimbo LM, Maklia EM, Salonen JJ, Laaksonen PH, Helariutta AM, Linder MB, Hirvonen JT, Laaksonen TJ, Santos HA, Airaksinen AJ, The mucoadhesive and gastroretentive properties of hydrophobin- coated porous silicon nanoparticles oral drug delivery systes. Biomaterials. 2012;33(11) 3353-62.

5. Dembinski A, Warzecha Z, Konturek SJ, Ceranowicz P, Dembinski M, Pawlik WW, Kusnierz- Cabala B, Naskalski JW, Extract of grapefruit- seed reduces acute pancreatitis induced by ischemia/ reperfusion in rats: possible implication of tissue antioxidants. J. Physiol. Pharmacol. 2004; 55(4) 811-2 1 .

6. Le Gall G, DuPont MS, Mellon A, Davis L, Collins GJ, Verhoeyen ME, Colquhoun IJ, Characterization and content of flavonoid glycosides in genetically modified tomato (Lycopersicon esculentum) fruits. J. Agric. Food Chem. 2003; 51(9) 2438- 46.

7. Wang H, Nair MG, Strasburg GM, Booren AM, Gray JI, Antioxidant polyphenols from tart cherries (Prunus cerasus). J. Agric. Food. Chem. 1999; 47(3) 840- 44.

8. Erlund I, Meririnne E, Alfthan G, Aro A, Plasma kinetics and urinary excretion of the flavonones naringenin and hesperitin in humans after ingestion of orange juice and grapefruit juice. J. Nutr. 2001; 131(2) 235- 41.

9. Stark T, Bareuther S, Hofmann T, Sensory- guided decomposition of roasted cocoa nibs (Theobroma cacao) and structure determination of taste- active polyphenols. J. Agric. Food. Chem. 2005; 53(13) 5407- 18.

10. Ekambaram G, Rajendran P, Magesh V, Sakthisekaran D, Naringenin reduces tumor size and weight loss in N-methylNnitro- N-nitrosoguanidine-induced gastric carcinogenesis in rats. Nutr Res. 2008; 28(2): 106- 112 .

11. Lauro MR, De Simone F, Sansone F, Lannelli P, Aquino RP, Preparations and release characteristics of naringin and naringenin gastro-resistant microparticles by spray-drying. J. Drug Del. Sci. Tech. 2007; 17: 119-124

12. Yen FL, Wu TH, Lin LT, Cham TM, Lin CC, NaringeninLoaded Nanoparticles Improve the Physicochemical Properties and the Hepatoprotective Effects of Naringenin in Orally-Administered Rats with CCl4-Induced Acute Liver Failure. Pharmaceutical Research. 2008;26(4) 893-902.

13. Harsha S, Dual drug delivery system for targeting H. pylori in the stomach: preparation and in vitro characterization of amoxicillin-loaded Carbopol ${ }^{\circledR}$ nanospheres. Int J Nanomedicine. 2012; 7: 4787- 4796.

14. Harsha S, Pharmaceutical suspension containing both immediate/sustained-release amoxicillin-loaded gelatin nanoparticles: preparation and in vitro characterization. Drug Design, Development and Therapy. 2013; 7: 1027- 1033.
15. Tu S, Wang BL, Chen YW, Li ZM, Luo XL, Polymeric nanocubes Spontaneously Formed from Poly ( $\varepsilon$ caprolactone). ACS Macro Lett. 2012; 1(8): 933-36.

16. Cheng D, Xia H, Chan HS, Fabrication of hollow nanospheres, hollow nanocubes and hollow plates. Nan otechno logy. 2006; 17: 1661- 67.

17. Slouf M, Hruby M, Bakaeva Z, Vlkova H, Nebesarova J, Philimonenko AA, Hozak P, Preparation of stable Pd nanocubes and their use in biological labeling. Colloids and Surfaces B: Biointerfaces. 2012; 100: 205- 8 .

18. Jain SK, Chourasia MK, Jain AK, Jain RK, Shrivastava AK, Envelopment and characterization of mucoadhesive microspheres bearing salbutamol for nasal delivery. Drug Deliv. 2004; 11: 113- 22.

19. Wang t, Jiang M, Wu Y, Nanoparticles composed of PLGA and hyperbranched poly (amine-ester) as a drug carrier. J Polym Res. 2010; 17:335-45.

20. Sofia P, Dimitrios B, Konstantinos A, Evangelos K, Manois $\mathrm{G}$, Chitosan nanoparticles loaded with dorzolamide and pramipexole. Carbohydrate Polymer. 2008; 73: 44- 54.

21. Zai J, Zhu J, Qi R, Qian X, Nearly monodispersed In (OH) hierarchical nanospheres and nanocubes: tunable ligandassisted synthesis and their conversion into hierarchical $\mathrm{In}_{2} \mathrm{O}_{3}$ for gas sensing. J. Mater. Chem. A. 2013; 1: 735- 45.

22. Schubert S, Delaney JT, Schubert US, Nanoprecipitation and nanoformulation of polymers: from history to powerful possibilities beyond poly (lactic acid). Soft Matter. 2010; 7: 1581-88.

23. Fessi H, Puisieux F, Devissaguet JP, Ammoury N, Benita S, Nanocapsule formulation by interfacial polymer deposition following solvent displacement. Int J Pharm. 1989; 55: R1R4.

24. Wang $\mathrm{T}$, Wang $\mathrm{X}$, LaMontagne $\mathrm{D}$, Wang $\mathrm{Z}$, Wang Z, Cao YC, Shape- Controlled Synthesis of Colloidal Superparticles from Nanocubes. J Am Chem Soc. 2012; 134: 18225- 28.

25. Hou $\mathrm{B}, \mathrm{Xu} \mathrm{Y}, \mathrm{Wu} \mathrm{D}$, Sun $\mathrm{Y}$, Size- controllable barium titanate nanopowder synthesized via one- pot solvothermal route in a mixed solvent. Powder Technology. 2006; 170: 127-33.

26. Huang L, Peng F, Yu H, Wang $\mathrm{H}$, Synthesis of $\mathrm{Cu}_{2} \mathrm{O}$ nanoboxes, nanocubes an $\mathrm{d}$ nanospheres by polyol process and their adsorption characteristic. Materials Research Bulletin. 2008; 43: 3047- 53.

27. Das S, Roy P, Auddy RG, Mukherjee A, Silymarin nanoparticle prevents paracetamol-induced hepatotoxicity. International Journal of Nanomedicine. 2011;6: 1291- 01 .

28. Horisawa E, Danjo K, Har una M, Physical properties of solid dispersion of a nonsteroidal anti-inflammatory drug (M-5011) with Eudra git E. Drug Dev. Ind. Pharm. 2000; 26: 1271- 78.

29. Jayasree J, Sivaneswari S, Hemalatha G, Preethi N, Mounika B, Vasudeva Murthy, Role of various natural, synthetic and semi- synthetic polymers on drug release kinetics of losartan potassium oral controlled release tablets. Int J Pharm Investig. 2014; 4(4) 183-88.

30. Alli SM, Ali SM, Samanta A, Development and evaluation of intestinal targeted mucoadhesive microspheres of Bacillus coagulans. Drug Dev Ind Pharm. 2011; 37 (11) 1329-38.

31. Tao Y, Lu Y, Sun Y, Gu B, Lu W, Pan J, Development of mucoadhesive microspheres of acyclovir with enhanced bioavailability. Int J Pharm. 2009; 378 (1-2) 30-6. 